\title{
New mouth cancer centre to improve survival and quality of life for thousands
}

A new cancer centre is being set up to improve survival rates and quality of life for thousands of people affected by squamous cancer - a specific type of cancer which affects the mouth, skin, lungs and cervix.

The centre, which will be based at Queen Mary University of London's Blizard Institute in Whitechapel, will bring together clinical and research experts to explore who is at risk of developing squamous cancer and why.

More than 70,000 people are diagnosed with squamous cancer every year in the UK. It is the most common cause of solid tumours and results in many deaths.

The Barts Centre for Squamous Cancer is being set up with a $£ 2.6$ million grant from Barts Charity, and will focus on mouth cancer as this is a particularly common problem among the local East London population.

Mouth cancer has increased by $58 \%$ over the past decade, yet despite more than 8,700 people being diagnosed every year across the UK, only one in five people know the main signs and symptoms.

Many patients will not survive for five years after being diagnosed, and for those who do, treatment can be harsh and disfiguring, often leaving a devastating impact on a person's appearance and ability to eat, drink and speak.

Smoking, alcohol, diet, and various viruses all increase an individual's risk of developing oral cancer, and it is a particular problem in areas of high social deprivation, and among certain groups, such as South Asian communities.

In the London Borough of Tower Hamlets, the rate has risen by a third over the past decade to 21.5 people per 100,000 , and it continues to increase among younger adults due to tobacco use.

Paul Coulthard, Professor of Oral \& Maxillofacial Surgery at Barts and The London School of Medicine and Dentistry at Queen Mary University of London, said: 'Oral cancer has been underfunded for many years, and we hope that by bringing our expertise together in this new centre we will be able to develop a better understanding of mouth cancer. Awareness of risk factors and symptoms is still very low, and we hope our work will improve detection, diagnosis, and access to treatment.

'We know that the risk of being diagnosed with oral cancer is strongly associated with social deprivation, and this is a particular health challenge in London. This centre will enable us to develop a much better understanding of who is at risk and why, so that we can improve treatment and the quality of life for all those affected, both in the UK and wider afield.'

The Barts Centre for Squamous Cancer will bring together clinical and research experts who will work with patient groups, run clinical trials, and build a human tissue bank to improve knowledge and understanding of squamous cancer. 